\title{
Accelerated Molecular Dynamics Simulations for Characterizing Plastic Deformation in Crystalline Materials with Cracks
}

\author{
Subhendu Chakraborty ${ }^{\mathrm{a}, 1}$, Jiaxi Zhang ${ }^{\mathrm{a}, 1}$, Somnath Ghosh ${ }^{\mathrm{a}, 2, *}$ \\ ${ }^{a}$ Departments of Civil, Mechanical, and Materials Science \& Engineering, Johns Hopkins \\ University, 3400 N. Charles Street, Baltimore, MD 21218
}

\begin{abstract}
Molecular Dynamics (MD) simulations are often used for comprehending evolving deformation mechanisms in materials at the atomic scale and also for assessing continuum-scale material properties. A major limitation of conventional MD simulations is that very small MD time-scale $(\sim f s)$, restrict the achievable strain-rates to be much higher $\left(\sim 10^{7}\right.$ or higher $)$ than experimentally observed rates, needed for continuum scale modeling, e.g. using crystal plasticity finite element methods. A strain-boost hyperdynamics method based accelerated MD tool is adopted and developed to overcome these limitations. This method biases the atomic system to make it evolve at much faster time-scales and achieve strain-rates that are at least three order of magnitude smaller than the lowest strain-rate achievable in MD. The hyperdynamics algorithm is implemented in a parallel version of LAMMPS, and validated with conventional MD. It is then used to predict evolution of plastic state variables at lower strain-rates for a Nickel single crystal with an embedded atomistic crack. It is shown that at lower strain-rates, not only the evolution of plastic variables are different, but for some configurations there is a shift in the plastic deformation mechanism from twin dominated to dislocation dominated.
\end{abstract}

Keywords: Accelerated molecular dynamics, Hyperdynamics, Crack tip

\footnotetext{
${ }^{*}$ Corresponding author: Tel: +1-410-516-7833; fax: +1-410-516-7473

Email address: sghosh20@jhu.edu (Somnath Ghosh )

${ }^{1}$ Graduate Research Associate

${ }^{2}$ M. G. Callas Professor
}

Preprint submitted to Elsevier

April 13, 2016

(C) 2016. This manuscript version is made available under the Elsevier user license

http://www.elsevier.com/open-access/userlicense/1.0/ 
plasticity, Nickel

\section{Introduction}

Deformation and failure behavior of metals inherently couple multiple spatial scales ranging from the atomic scale of defects and deformation mechanisms such as dislocations and twins to the continuum domains of grains and polycrystalline aggregates. Modeling the evolution of defects such as cracks and voids, their associated deformation mechanisms and interactions, often requires understanding and representation at the atomistic scales. Spatial and temporal coarse-graining of the atomistic behavior are subsequently needed to propose plasticity and damage constitutive relations at the continuum scale of single crystals. Molecular dynamics (MD) is one of the most effective simulation tools currently in use to investigate the behavior of many materials at atomistic scale. With increasing computational power, large scale atomistic simulations are routinely being conducted with several million or even billion atom systems, e.g. in $[12,40]$ to study deformation and fracture in metallic systems. MD simulations have been used to study deformation mechanisms due to crack tip plasticity at finite temperatures in $[37,38]$. In [39] simulations have been conducted at very low temperatures, thus eliminating any thermal activation and investigating only stress driven processes.

MD simulations used to study fatigue and failure are often faced with serious limitations in realizing experimental strain-rates. The typically achieved MD time-scales are in the nano- (ns) to micro-seconds ( $\mu s)$ range, corresponding to atomic vibrations with time-period in the order of pico-seconds (ps). Temporal resolutions required in the solution of the dynamical systems using incremental time-integration algorithms, limit the time-steps to be of the order of femto-seconds $\left(f_{s}\right)$. Even with today's powerful computing platforms, this limits the maximum physical time to the micro-second ( $\mu s)$ range. Correspondingly, in the study of deformation mechanisms in a deformable body, one is compelled to use very high strain-rates of $\sim 10^{7}$ or even higher to achieve strains of any physical significance. Thermal activation of stress-driven processes like nucleation of dislocations and microtwins make the mechanical behavior of materials temperature and strain-rate dependent. Very high strain-rates in conventional MD simulations can result in very different activation regimes compared to those in laboratory experiments. This 
leads to the activation of different temperature and strain-rate dependent deformation mechanisms like surface mediated dislocation nucleation [41]. High strain rate based MD simulations of fcc single crystal containing a crack in a certain orientation predicts a twin dominated deformation under mode-I loading, whereas room temperature experiments under similar loading do not show any twinning. This anomaly between experimental and simulation results has been attributed to the strain-rate effects in [36] where it has been shown that there is a transition from crack-tip twining at short times to full dislocation formation at long times.

Various acceleration methods have been proposed in the literature to mitigate limitations of extremely small time-scales in MD simulations. These include the parallel replica dynamics or PRD method [30, 35], the temperature accelerated dynamics or TAD method [27], and the hyperdynamics [33]. The hyperdynamics is one of the most effective time acceleration methods for predicting the temporal evolution of atomic systems. It has been used to study thermally activated nucleation processes like adaton diffusion on a substrate in [33] and also to investigate the stress driven thermally activated process like dislocation nucleation from sharp corners of a nanopillar in [8] and an atomic crack-tip in [3].

In the present work, the hyperdynamics-based accelerated MD method has been extended beyond the nucleation events to study deformation mechanisms in the plastic regime and quantify the evolution of plastic variables, which can be used in continuum based material models such as dislocation density-based crystal plasticity. The structure of the paper is as follows. A brief description of different time-scale acceleration methods are given in section 2. In section 3 different aspects of the hyperdynamics method are discussed with special focus on strain-boost hyperdynamics. Particular emphasis is on the construction of a boost potential with evolving parameters, as well as their implementation and validation. Finally in section 5.2, a comparative study of the strain-rate effect is presented using high strain-rate conventional MD and low strain-rate hyperdynamics-based accelerated MD simulations. 


\section{Methods of Time-scale Accelerated Molecular Dynamics Simu- lations}

Various methods have been developed in the literature to achieve longer physical time-scales in MD simulations. A few of the important methods suitable for time acceleration of driven solid state systems are briefly discussed in this section.

\subsection{The Parallel Replica Dynamics (PRD)}

The parallel replica dynamics or PRD method [35] is a simple yet accurate method that is based on the fact that most atomic processes are statistical in nature. It is based on the assumption that ensemble averaging over more micro-states is equivalent to exploring the system for longer time durations. This is achieved by temporal parallelization of the simulations over multiple processors, where individual processors run a replica of the sample independent of each other. This is in contrast with conventional parallelization, where the computational model is spatially distributed among multiple processors. The total number of micro-states explored in the PRD simulation process is sum from all the processors. Hence the accumulated simulation time $\left(t_{p h y}\right)$ is related to MD time of the individual $i-t h$ replica $\left(t_{M D}^{i}\right)$ as:

$$
t_{p h y}=\sum_{i=1}^{i=S} t_{M D}^{i} \approx S t_{M D}
$$

where $\mathrm{S}$ is the total number of replicas and $t_{M D}$ is the average time. It is obvious that the level of acceleration that can be achieved is approximately proportional to the number of processors (or replicas) used.

\subsection{The Temperature Accelerated Dynamics (TAD)}

The temperature accelerated dynamics (TAD) [27] is a method of accelerating the time evolution of an atomic system, based on the observation that at finite temperatures an atomic system spends a substantial amount of time in the local potential well. Individual atoms vibrate with respect to their equilibrium position until an atom or a cluster of atoms acquire sufficient energy to overcome the local energy barrier and move to an adjacent potential well. The process continues until the system finds the global potential well. Material behavior is controlled by processes that are mainly stress driven and/or thermally activated. Thermally activated processes, 
which correspond to transition of a system from one local potential well to another, are inherently temperature dependent. Thus, the rate of this transition increases with temperature as the thermal energy available to overcome the energy barrier increases. To accelerate these transitions, simulations are performed at elevated temperatures but also by eliminating any spurious transition that does not occur at the original temperature. The speed-up or boost $S U_{T A D}$ that is achieved by this method is expressed as:

$$
S U_{T A D}=\exp \left[E_{\min }\left(\frac{1}{K_{b} T_{\text {low }}}-\frac{1}{K_{b} T_{\text {high }}}\right)\right]
$$

where $E_{\min }$ is the minimum energy barrier for state to state transition, $T_{\text {low }}$ is the temperature at which the original MD simulation is intended, $T_{\text {high }}$ is the elevated temperature at which the MD simulation is actually performed, and $K_{b}$ is Boltzmann constant. A major challenge with this method is to come up with an appropriate criterion for filtering out the spurious transition, and extrapolating the state to state transition rate from an elevated temperature $T_{\text {high }}$ to a lower one $T_{\text {low }}$.

\subsection{Hyperdynamics (HD)}

A very efficient way of accelerating the transition from one potential well to another for an atomic system had been proposed with the hyperdynamics or (HD) method in [34]. This method is based on lifting the basin of the potential-well or biasing the local potential landscape as shown in fig. 1). A boost potential is added to the original system potential shown with the solid line to lift the potential well. The biased potential shown with the dashed line makes state to state transition more frequent i.e, at an accelerated pace. Time evolution of the biased system and the unbiased system can be related using the Transition State Theory (TST) [6, 29, 31]. From the TST and equilibrium statistical mechanics based ensemble-averaging it can be shown that time evolution of the atomic system under biased potential is related to the MD time step as:

$$
\Delta t_{p h y}=\Delta t_{M D} \exp \frac{\Delta V_{b}}{K_{b} T}
$$

where $\Delta t_{p h y}$ is the actual time evolved for an incremental MD time of $\Delta t_{M D}$, $\Delta V_{b}$ is the boost potential applied to bias the system, $T$ is simulation temperature and $K_{b}$ is Boltzmann constant. A comprehensive mathematical construction of this method may be found in [16, 34]. 


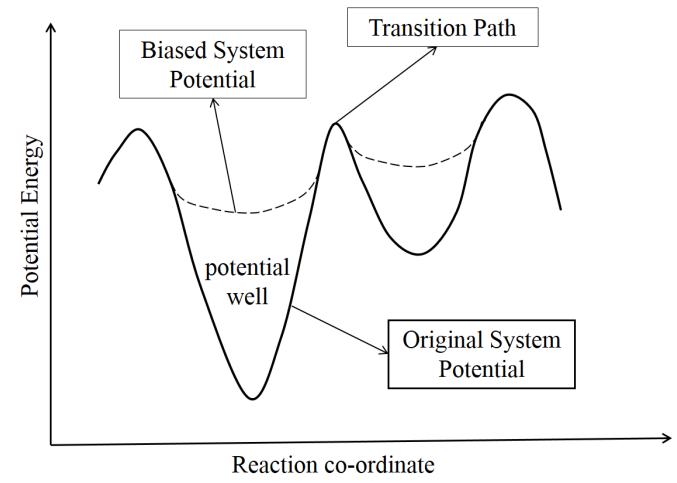

Figure 1: Schematic illustration of the hyperdynamics method. The solid line corresponds to the original system potential, while the biased potential is shown with the dashed line.

The most challenging task in this method is to construct the boost potential that will provide sufficient time acceleration, consistent with the TST. A general form of the boost potential is given as:

$$
\Delta V(\mathbf{r})=\frac{F}{N_{b}} \sum \delta V_{i}
$$

where $\delta V_{i}$ is the boost potential applied to the $i-t h$ atom and $N_{b}$ is the total number of atoms to be boosted. $F$ is a stopping function that will drive the boost potential to zero as the system approaches the dividing surface, such that the assumption of hyperdynamics will not be violated. A number of methods have been proposed to construct appropriate boost potentials that will provide sufficient boost with minimal computational cost, e.g. in $[8,11,20]$. A strain-boost hyperdynamics formulation, proposed in [8], is adopted in the present study. In this model, the second invariant of the deviatoric local atomic strain (addressed as the von-Mises shear strain invariant) is used to construct the boost potential. A major advantage of this approach is that the local atomic strain is a bond angle sensitive local geometric variable, which better reflects the condition of the nearest-neighbor atomic shell than the bond length alone [8]. This method is discussed in sec. 3.

Other sophisticated methods have also been developed for more complicated systems. Among these, the most widely used are metadynamics 
[17, 18], thermodynamics integration [5], umbrella sampling [13, 14], multicanonical ensemble [4]etc. These methods are most suitable to study more complicated systems, e.g, reaction mechanism of different molecular systems in the liquid phase, conformational changes of long molecular chains in solution, protein folding, protein-protein interaction, phase transition etc. In metadynamics, the system is adaptively biased by iterative injection of a small Gaussian potential, which is a function of carefully chosen collective state variables. A major advantage of this method is that history dependent bias prevents the system from visiting those part of the phase-space, which it has already explored. By injecting a smaller Gaussian potential successively, the free energy of the system can be estimated with high accuracy. In thermodynamics integration [5] the transition is accelerated by constraining the reaction coordinates at different values in multiple windows and forcing the system to sample along a line perpendicular to the reaction coordinate. Special care has to be taken to constrain the reaction coordinate in an energy-conserving manner. Contrary to thermodynamics integration, in umbrella sampling [13] the reaction coordinate is not constrained in multiple windows, but rather pulled to a target value by using appropriate bias potential [14].

These methods will be most suitable if the free energy of the system is not known a priori. Such cases arise when the system of interest is complicated and transition of interest is preceded by many non-interesting events manifested by local potential wells. In contrast, the problem investigated here includes plastic deformation of crystalline material, where the event of interest is very precise i.e, nucleation of dislocations. The energy barrier corresponding to that transition can be estimated by some trial MD simulation with much smaller system size. This is discussed in section 3.2.

\section{Strain Boost Hyperdynamics}

The boost potential used in the present study is a function of the second invariant of the least-square atomic strain, as given in [42]. With this augmentation, equation (4) takes the form: 


$$
\begin{aligned}
\Delta V(\mathbf{r}) & =\frac{F\left(\eta_{\text {max }}^{\text {Mises }}\right)}{N_{b}} \sum \delta V_{i}\left(\eta_{i}^{\text {Mises }}\right) \quad \text { where } \\
\delta V_{i}\left(\eta_{i}^{\text {Mises }}\right) & =V_{\max }\left[1-\left(\frac{\eta_{i}^{\text {Mises }}}{q_{c}^{\text {max }}}\right)^{2}\right]
\end{aligned}
$$

Here $\eta_{i}^{\text {Mises }}$ is second invariant of the local atomic strain $\eta_{i}$ of the $i-t h$ atom and $\eta_{\text {max }}^{\text {Mises }}=\max \left\{\eta_{i}^{\text {Mises }}, i=1,2, \ldots, N_{b}\right\}$, where $N_{b}$ is the number of boost atoms. $\delta V_{i}$ is boost potential, which is a function of $\eta_{i}^{\text {Mises }}(\mathbf{r})$. $F\left(\eta_{\text {max }}^{\text {Mises }}\right)$ is the stopping function that enforces the hyperdynamics assumptions on the boost potential at transition. The following form of stopping function is used in this study.

$$
F\left(\eta_{\text {max }}^{\text {Mises }}\right)= \begin{cases}1-\left(\frac{\eta_{\text {max }}^{\text {Mises }}}{q_{c}^{\text {max }}}\right)^{2}, & \forall \eta_{\text {max }}^{\text {Mises }}<q_{c}^{\text {max }} \\ 0 & \forall \eta_{\text {max }}^{\text {Mises }} \geq q_{c}^{\text {max }}\end{cases}
$$

The cardinal ingredient in constructing both the boost potential and stopping function is the atomic strain. The procedure to compute local atomic strains from given atomic configurations is discussed next. Consider two configuration of an atomic system, one at time $t=0$ denoted as the reference configuration $\mathbf{x}_{\mathbf{i}}^{\mathbf{0}}$ and other at time $t$ designated as the current configuration $\mathbf{x}_{\mathbf{i}}$. The separation vector of an atom $i$ with it's neighbor $j$ in the reference configuration and current configuration are respectively written as:

$$
\mathbf{d}_{j i}^{0}=\left(\mathbf{x}_{j}^{0}-\mathbf{x}_{i}^{0}\right) \quad \text { and } \quad \mathbf{d}_{j i}=\left(\mathbf{x}_{j}-\mathbf{x}_{i}\right)
$$

For this discrete system, the objective is to realize a function $\mathbf{J}_{i}$ that maps the vector from the reference to current configuration, i.e.

$$
\mathbf{d}_{j i}^{0} \stackrel{\mathbf{J}_{i}}{\rightarrow} \mathbf{d}_{j i} \quad \forall \quad j \in N_{i}
$$

where $N_{i}$ is the set of all neighbors of $i-t h$ atom. In [42] the function $\mathbf{J}_{i}$ is determined in a least-square sense by minimizing the total mapping error $\left(\sum_{j=1}^{N_{i}}\left(\mathbf{d}_{j i}^{0} \mathbf{J}_{i}-\mathbf{d}_{j i}\right)^{2}\right)$. This minimization leads to a functional form of tbe 
deformation gradient for a discrete system as:

$$
\mathbf{J}_{i}=\mathbf{V}_{i}^{-1} \mathbf{W}_{i}
$$

where

$$
\mathbf{V}_{i}=\sum_{j=1}^{N_{i}} \mathbf{d}_{j i}^{0 T} \mathbf{d}_{j i}^{0} \quad \text { and } \quad \mathbf{W}_{i}=\sum_{j=1}^{N_{i}} \mathbf{d}_{j i}^{0 T} \mathbf{d}_{j i}
$$

Upon evaluation of the deformation gradient $\mathbf{J}_{i}$ matrix, the Lagrangian strain $\boldsymbol{\eta}_{i}$ for a discrete system may be constructed as:

$$
\boldsymbol{\eta}_{i} \equiv\left(\mathbf{J}_{i} \mathbf{J}_{i}^{T}-\mathbf{I}\right)
$$

where $\mathbf{I}$ is the identity matrix. For a frame-invariant boost potential that is independent of reference frame, its construction uses the second invariant of the deviatoric part of $\boldsymbol{\eta}_{i}$, given as:

$$
\boldsymbol{\eta}_{i}^{\text {Mises }} \equiv \sqrt{\frac{1}{2} \operatorname{Tr}\left(\boldsymbol{\eta}_{i}-\eta_{i}^{\text {hydro }} \mathbf{I}\right)^{2}}
$$

where $\operatorname{Tr}$ is the trace and $\eta_{i}^{\text {hydro }}$ is the hydrostatic part of the $\boldsymbol{\eta}_{i}$. The atomic strain-based boost potential construction requires two parameters $V_{\max }$ and $q_{c}^{\max }$ in equations (5) and (6). Details on how to calculate these parameters for a specific material are discussed in sections 3.2 and 3.3.

An important prerequisite of hyperdynamics is to make the boost potential $\delta V_{i}$ go to zero on all dividing surfaces, as the system approaches a saddle point for the transition from one local potential well to another as shown in figure 1. A method of finding these saddle points of the potential surface is by calculating the gradient vector $g_{i}\left(=\partial V / \partial x_{i}\right)$ and the Hessian matrix $H_{i j}\left(=\partial^{2} V / \delta x_{i} \partial x_{j}\right)$. Here $\mathbf{x}$ is $3 \mathrm{~N}$ dimensional vector where $\mathrm{N}$ is the number of atoms in the system [34]. Solving for $g_{i}=0$ will give all the extremums of the energy surface. Of these points, the one for which the Hessian matrix has one negative eigenvalue belongs to the saddle point. However, a problem with this approach is that the energy surface is not known a-priori. Additionally, finding all solutions of $g_{i}=0$ for a $3 N$ dimensional potential energy function, and the eigenvalues of the corresponding Hessian matrix $H_{i j}$ is a computationally expensive process and almost intractable for large systems. 
A consideration used to overcome this problem is that, whenever a system passes through the saddle point for a transition from one local potential well to another, it undergoes some major configurational change in the atomic system involving at least one atom and it's nearest neighbors. Consequently, any state variable that reflects this configurational change can be used as an indicator of the transition. The critical value of this indicator can be used as a threshold beyond which the boost potential will be forced to zero. In the present study, the stopping function $F\left(\eta_{\text {max }}^{\text {Mises }}\right)$ in equation (6) serves this purpose, where $q_{c}^{\max }$ is the critical value of $\eta_{\max }^{\text {Mises }}$. Figure 2 shows the evolution of potential energy and $\eta^{\text {Mises }}$ at $\mathrm{T}=2 \mathrm{~K}$ for a typical atom in the ensemble during the nucleation of a leading partial dislocation at approximately $252 \mathrm{ps}$ into the simulation.
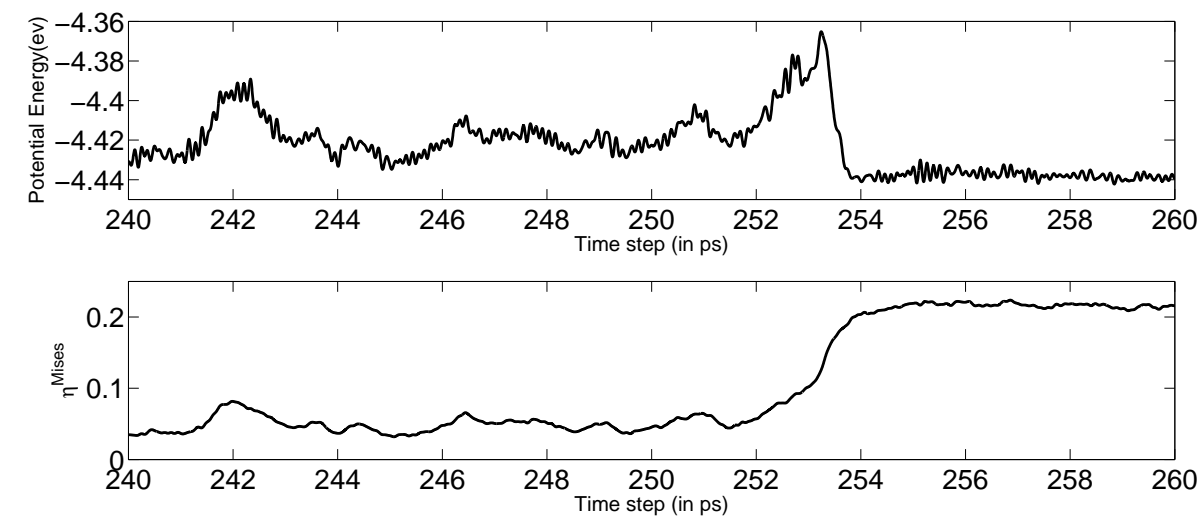

Figure 2: Time evolution of potential energy and $\eta^{\text {Mises }}$ of a typical atom in the ensemble at $T=2 K$.

Substantial changes in the magnitude of $\eta^{\text {Mises }}$ at the onset of nucleation makes it a perfect indicator of the transition. An alternate indicator, proposed for bond-boost hyperdynamics in [20], is the critical bond length. Whenever a tagged bond crosses some critical length, the system is considered to be on the verge of a transition from the current potential well to an adjacent one. In the present analysis, the aim is to investigate dislocation dominated crack tip plasticity. For this problem, the strain-boost model is chosen over the bond-boost method since dislocation nucleation is a collective behavior of an atomic cluster. The local atomic strain is a better collective variable, which involves at least one atom and it's nearest neigh- 
bors in comparison with the maximum bond length between an atom and it's neighbor. Noteworthy in this discussion is the fact that twin nucleation and twin-boundary propagation involves sequential nucleation of leading partials on parallel slip planes.

\subsection{Hyperdynamics for driven systems}

The original development of hyperdynamics in [33] was for non-driven systems, where the potential energy of the system and energy barrier between states remain unchanged. This renders the TST rate to remain constant throughout the simulation for both the biased and the original systems. The ratio of these two rate constants is interpreted as the acceleration in timescale due to the applied bias and is quantified in the form of a boost factor. For a driven system examined in present study, the TST rate at two different strain states are different. However for a carefully chosen boost potential, the ratio of the TST rate for the biased system and the original system can remain unchanged throughout the simulation. This means that the applied macroscopic strain influences the TST rate of both the biased and original systems in same manner. This implies that for two consecutive time steps $t_{1}$ and $t_{2}$ with macroscopic strains $\epsilon_{1}$ and $\epsilon_{2}$, even though

$$
\left.K_{A \rightarrow B}^{T S T}\right|_{\epsilon_{1}} \neq\left. K_{A \rightarrow B}^{T S T}\right|_{\epsilon_{2}} \quad \text { and }\left.\quad K_{A_{b} \rightarrow B}^{T S T}\right|_{\epsilon_{1}} \neq\left. K_{A_{b} \rightarrow B}^{T S T}\right|_{\epsilon_{2}}
$$

the following relation still holds:

$$
\left.\left.\frac{K_{A_{b} \rightarrow B}^{T S T}}{K_{A \rightarrow B}^{T S T}}\right|_{\epsilon_{1}} \approx \frac{K_{A_{b} \rightarrow B}^{T S T}}{K_{A \rightarrow B}^{T S T}}\right|_{\epsilon_{2}}
$$

The notations used have the same meaning as in [33]. Consequently, the criterion to be satisfied is,

$$
\left.\frac{K_{A_{b} \rightarrow B}^{T S T}}{K_{A \rightarrow B}^{T S T}}\right|_{\epsilon_{1}}=\left.\frac{K_{A_{b} \rightarrow B}^{T S T}}{K_{A \rightarrow B}^{T S T}}\right|_{\epsilon_{2}}
$$


From the relations in [33],

$$
K_{A \rightarrow B}^{T S T}=\frac{\left\langle\left|v_{A}\right| \delta_{A}(\mathbf{r})\right\rangle_{A_{b}}}{\left\langle e^{\beta \Delta V_{b}(\mathbf{r})}\right\rangle_{A_{b}}}
$$

The numerator in the right hand side is the TST rate constant under a biased potential. This renders equation (16) to be

$$
K_{A \rightarrow B}^{T S T}=\frac{K_{A_{b} \rightarrow B}^{T S T}}{\left\langle e^{\beta \Delta V_{b}(\mathbf{r})}\right\rangle_{A_{b}}}, \Longrightarrow \frac{K_{A_{b} \rightarrow B}^{T S T}}{K_{A \rightarrow B}^{T S T}}=\left\langle e^{\beta \Delta V_{b}(\mathbf{r})}\right\rangle_{A_{b}}
$$

Substituting equation (17) in equation (15) yields:

$$
\left.\left\langle e^{\beta \Delta V_{b}(\mathbf{r})}\right\rangle_{A_{b}}\right|_{\epsilon_{1}}=\left.\left\langle e^{\beta \Delta V_{b}(\mathbf{r})}\right\rangle_{A_{b}}\right|_{\epsilon_{2}}
$$

For constant temperature, the parameter $\beta\left(=\frac{1}{K_{B} T}\right)$ remains unchanged throughout the simulation. So, for equation (18) and consequently equation (15) to hold, the applied boost potential has to be independent of macroscopic strain $\epsilon$, i.e.

$$
\left.\Delta V_{b}(\mathbf{r})\right|_{\epsilon_{1}}=\left.\Delta V_{b}(\mathbf{r})\right|_{\epsilon_{2}}
$$

In the present study it is observed that the effect of the macroscopic strain is more prominent on $\eta_{\text {max }}^{\text {Mises }}$ than on $\eta_{i}^{\text {Mises }}$. For the strain boost hyperdynamics, the effect of $\eta_{\max }^{\text {Mises }}$ on the boost potential comes through the construction of a stopping function given in equations (5) and (6). To mitigate this effect of macroscopic strain, the parameter $V_{\max }$ is monitored using the value of stopping function to keep the strength of the bias close to the activation free energy of nucleation. This procedure is discussed in details in section 3.2 .

It is also noteworthy that classical statistical mechanics-based ensemble averaging, which is used to relate the MD-time $\left(\Delta t_{M D}\right)$ with physical time $\left(\Delta t_{p h y}\right)$, (see equation (3)), can only be applied for thermally equilibrated systems. However, many problems of interest in mechanics of materials are not only thermally activated, but stress driven as well. One prime example 
is the nucleation of dislocations or gliding of existing dislocations, when a critical resolved shear stress is exceeded. One approach to keep the system near thermal equilibrium is to use a stepped loading in lieu of continuous ramped loading [15] and apply hyperdynamics using the instantaneous nearequilibrium system potential corresponding to the non-driven segment of the loading. In the present study, the system is far from equilibrium only when dislocation nucleates and continues until it reaches a stable configuration, or when dislocations in a stable configuration start gliding again due to the buildup of resolved shear stress to a critical value. During these far from equilibrium system-states, hyperdynamics is not activated, to comply with the methods basic assumptions. Other than these two non-equilibrium scenarios, the system mostly deforms elastically under quasi-equilibrium conditions. Furthermore, the system is forced to near-equilibrium states by imposing the macroscopic strain using affine transformation.

\subsection{Determination of $V_{\max }$}

The parameter $V_{\max }$ in equation (5b) is an important determinant of the maximum achievable boost and in turn temporal acceleration of the MD simulations. It should be high enough to give sufficient boost, while not creating any extra local potential well of significant depth. Rearranging equations (5a), (5b) and (6) yields,

$$
\begin{array}{rlrl}
\Delta V(\mathbf{r}) & =\frac{S}{N_{b}} \sum\left[1-\left(\frac{\eta_{i}^{\text {Mises }}}{q_{c}^{\text {max }}}\right)^{2}\right] & & \forall \eta_{\text {max }}^{\text {Mises }}<q_{c}^{\text {max }} \\
& =0 & \forall \eta_{\text {max }}^{\text {Mises }} \geq q_{c}^{\text {max }}
\end{array}
$$

where,

$$
S=V_{\max }\left[1-\left(\frac{\eta_{\max }^{\text {Mises }}}{q_{c}^{\max }}\right)^{2}\right]
$$

$S$ is termed as strength of the bias. In general, at the onset of transition, $S$ should be of the order of the potential barrier height that an atom has to overcome to initiate any nucleation. This will also guarantee that there will be no extra potential well created inside the original one near the dividing surface. A qualitative assessment of this barrier height $(0.2 \mathrm{ev}$ for present study at $300 \mathrm{~K}$ ) can be obtained by monitoring the time evolution of potential energy of a typical atom as it participates in the nucleation process, as shown 
in figure 3. It can be seen that after repeated failed attempts for which $\eta^{\text {Mises }}$ touches the $\eta_{\text {max }}^{\text {Mises }}=0.9 q_{c}^{\max }$ line, the atom acquires sufficient thermal energy at $\approx 324 p s$ to overcome the potential barrier and nucleate a leading partial dislocation. Every material has its own characteristic potential barrier height corresponding to the event of interest. Once the barrier height is obtained, $V_{\max }$ can be calculated using equation (21) as:

$$
V_{\max }=\frac{S}{\left[1-\left(\frac{\eta_{\max }^{\text {Mises }}}{q_{c}^{\text {max }}}\right)^{2}\right]}
$$

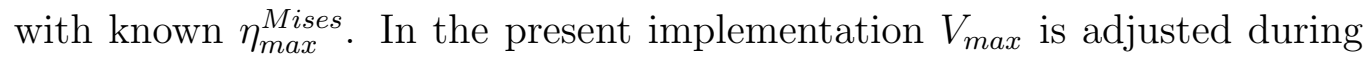
the simulation based on the average value of $\eta_{\text {max }}^{\text {Mises }}$. The running time av-

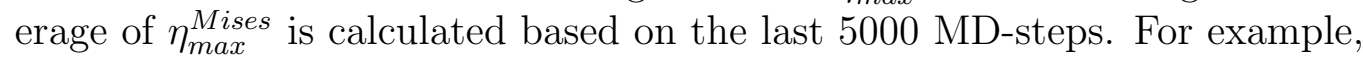
if the average $\eta_{\text {max }}^{\text {Mises }}$ is $0.5 q_{c}^{\max }$, for the strength of bias $(S)$ to be same as the potential barrier height i.e, $0.2 \mathrm{ev}, V_{\max }$ is set to be 0.2667 . This process of continuous monitoring of $V_{\max }$ guarantees that the instantaneous boost potential will never exceed the activation free energy of nucleation. It is noteworthy that $\eta_{\max }^{\text {Mises }}$ is the maximum value of $\eta^{\text {Mises }}$ among all atoms that are boosted. Of these atoms, the identity of the most critical atom keeps changing with the progress of simulation. Figure 3 shows the time evolution of potential energy and $\eta^{\text {Mises }}$ of one such atom. For the system studied, it is observed that at the onset of transition, the critical atoms frequently visit the state corresponding to $\eta^{\text {Mises }} \sim 0.9 q_{c}^{\max }$. The instantaneous boost potential for that particular atom, corresponding to this state, is $0.0380 \mathrm{ev}$ (from equation (20) with $S=0.2 \mathrm{ev}$ ).

Determination of the optimal $V_{\max }$ is crucial. Higher $V_{\max }$ will lead to a wrong conversion of the MD time $\left(\Delta t_{M D}\right)$ to the physical time $\left(\Delta t_{p h y}\right)$. In general, the strength of bias $S$ should be similar to the activation free energy of nucleation. The present implementation enforces this condition by adjusting $V_{\max }$ during the simulation. Another way to make this adaptive adjustment is by monitoring the frequency at which the identity of the most critical atom changes. This makes the requirement of prior knowledge of the energy barrier redundant. A more detailed description of the procedure with application on bond-boost hyperdynamics (also called Self-learning Bond Boost method) can be found in [24, 25]. 

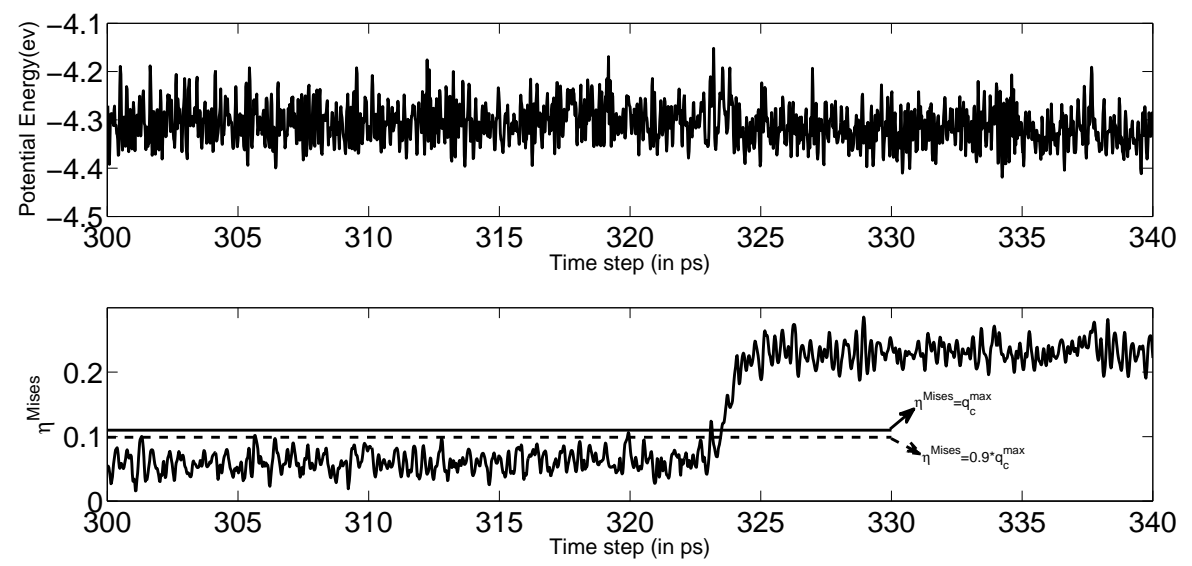

Figure 3: Time evolution of potential energy and $\eta^{\text {Mises }}$ of a typical atom in the ensemble at $T=300 K$.

Determination of $S$ and hence $V_{\max }$, sometimes can be challenging if the free energy corresponding to the event of interest is not known apriori. This situation arises when hyperdynamics itself is needed for the occurrence of those infrequent events in atomistic simulations such as in atomic diffusion in solids. A better approach for such situations is to use metadynamics $[17,18]$ or more sophisticated adaptive-boost MD [1] to get an approximate idea of the potential barrier height with little additional computational cost.

\subsection{Determination of $q_{c}$}

To find the optimal value of the threshold parameter $q_{c}^{\max }$, MD simulations are performed for a small atomistic ensemble with an embedded crack (created by removing few layers of atoms) to facilitate inhomogeneous nucleation of crack-tip dislocations. The parameter $q_{c}^{\max }$ indicates that the system is approaching a transition point, even without any additional thermal vibration. Hyperdynamics must stop beyond that point, i.e. the boost potential should go to zero to comply with it's assumption. $q_{c}^{\max }$ is inherently dependent on temperature due to the contribution from thermal vibration of the atomic system. An iterative technique is used in this study to find the optimal value of $q_{c}^{\max }$ at a particular temperature.

The potential energy of the system is first obtained for two different temperatures, one being very low ( $2 \mathrm{~K}$ for the present study) and the other corre- 
sponding to the temperature at which $q_{c}^{\max }$ is expected. The boost potential is then applied and the simulations culminating in system quenching are run corresponding to different assumed trial parameter values $q_{c}^{\text {trial }}$. If $q_{c}^{\text {trial }}$ is equal to the critical threshold value $q_{c}$ then the system will make a transition without any additional thermal fluctuation. The equilibrated potential energy determines whether the quenched system has made this transition, as elucidated in figure 4 . For $q_{c}^{\text {trial }}=0.1$ the system does not make the transition and falls back to the potential energy corresponding to the lower temperature pathway $2 \mathrm{~K}$. However transition takes place for $q_{c}^{\text {trial }}=0.12$ and $q_{c}^{\text {trial }}=0.13$. To obtain the minimum value of $q_{c}^{\text {trial }}$ for which transition takes place, the quench-simulation process is repeated with different initial atomic configurations by dephasing. The critical $q_{c}$ is assessed from this exercise. In this study, the parameter $q_{c}^{\text {trial }}$ is gradually incremented from 0.8 to 0.15 with a step size of 0.001 , and the critical value is found to be $q_{c}=0.12$ at $300 \mathrm{~K}$. Any value of $q_{c}^{\max }$ less than this critical $q_{c}$ is safe. A conservative value of $q_{c}^{\max }=0.11$ is used for all subsequent studies. A value of $q_{c}=0.095$ at $200 \mathrm{~K}$ has been used in [8] for the study of dislocation nucleation in copper nanopillars under compressive loading.

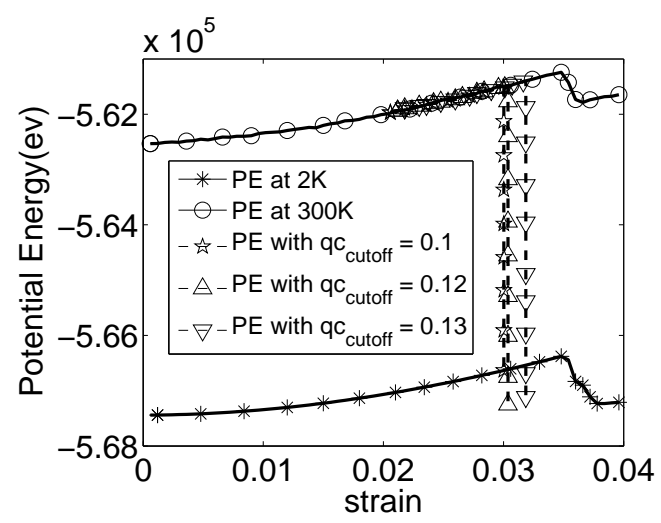

Figure 4: Plot of the potential energy as a function of applied strain corresponding to different temperatures in the quenching bath for determining $q_{c}$.

\subsection{Determination of $N_{b}$}

The number of atoms $N_{b}$ that need to be boosted in equation (5a) is another important parameter to be determined. The calculation of boost potential and corresponding forces involve some computational costs per 
atom. Hence, computational efficiency requires an optimally low number of atoms to be boosted while making sure to include the cluster of atoms that is most critical for the nucleation process. Since this paper focuses on the investigation of plastic deformation of a nickel single crystal in the presence of an atomistic crack, the crack tip region is the most critical part for nucleation. Consequently at the start of simulations, only a small throughthickness cylindrical domain with its center at the crack tip is boosted. This is demonstrated in figure 5. Based on potential energy, additional atoms are tagged as to-be-boosted, as the material deforms plastically. It should be noted that since the least-squares based deformation gradient is not a good measure of the local atomic strain for atoms with a low near-neighbor count, atoms with less than 12 first nearest neighbors for fcc crystals are not boosted in this work.

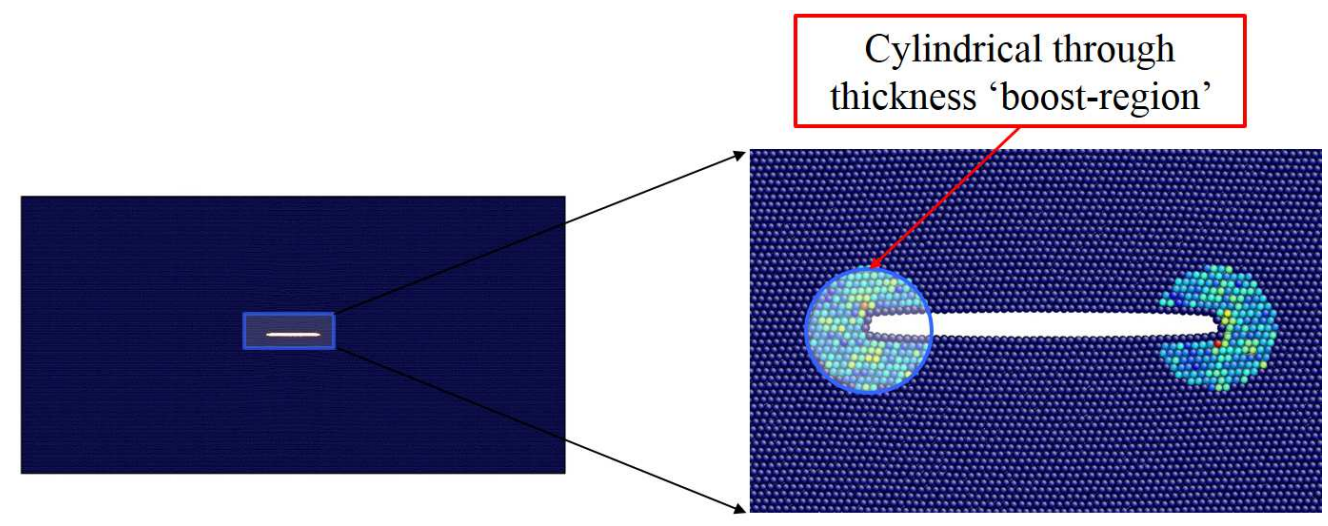

Figure 5: (a) A 3D atomic domain of single crystal nickel with an embedded crack for MD simulations, and (b) zoomed in view of the boost region for HD based accelerated MD simulations. The color of atoms is based on the second invariant of atomic strain $\left(\eta_{i}^{\text {Mises }}\right)$ corresponding to $1 \%$ macroscopic strain.

\section{Implementation of the Strain-Boost Hyperdynamics in LAMMPS and Accelerated MD Simulations}

The strain boost- based hyperdynamics method is implemented in a parallel version of the popular MD simulation code Large-scale Atomic/Molecular Massively Parallel Simulator or LAMMPS [26]. To keep the main structure of the LAMMPS unchanged, an additional "compute" module is incorporated. For a given reference and current configuration, this module calculates (i) 
the local least-squares based atomic-strain per atom, (ii) the boost potential per atom, and (iii) the additional force due to the boost potential per atom. For preliminary verification of this implementation, an MD model specimen is created in LAMMPS and subsequently deformed by using the "fix-deform" option without performing any time integration, thus eliminating any noise due to thermal vibration. The least squares based deformation gradient is computed using equation (9) for three different load cases viz. pure translation, pure rotation and pure stretch. The computed values are in agreement with the known deformation gradients for these deformation modes.

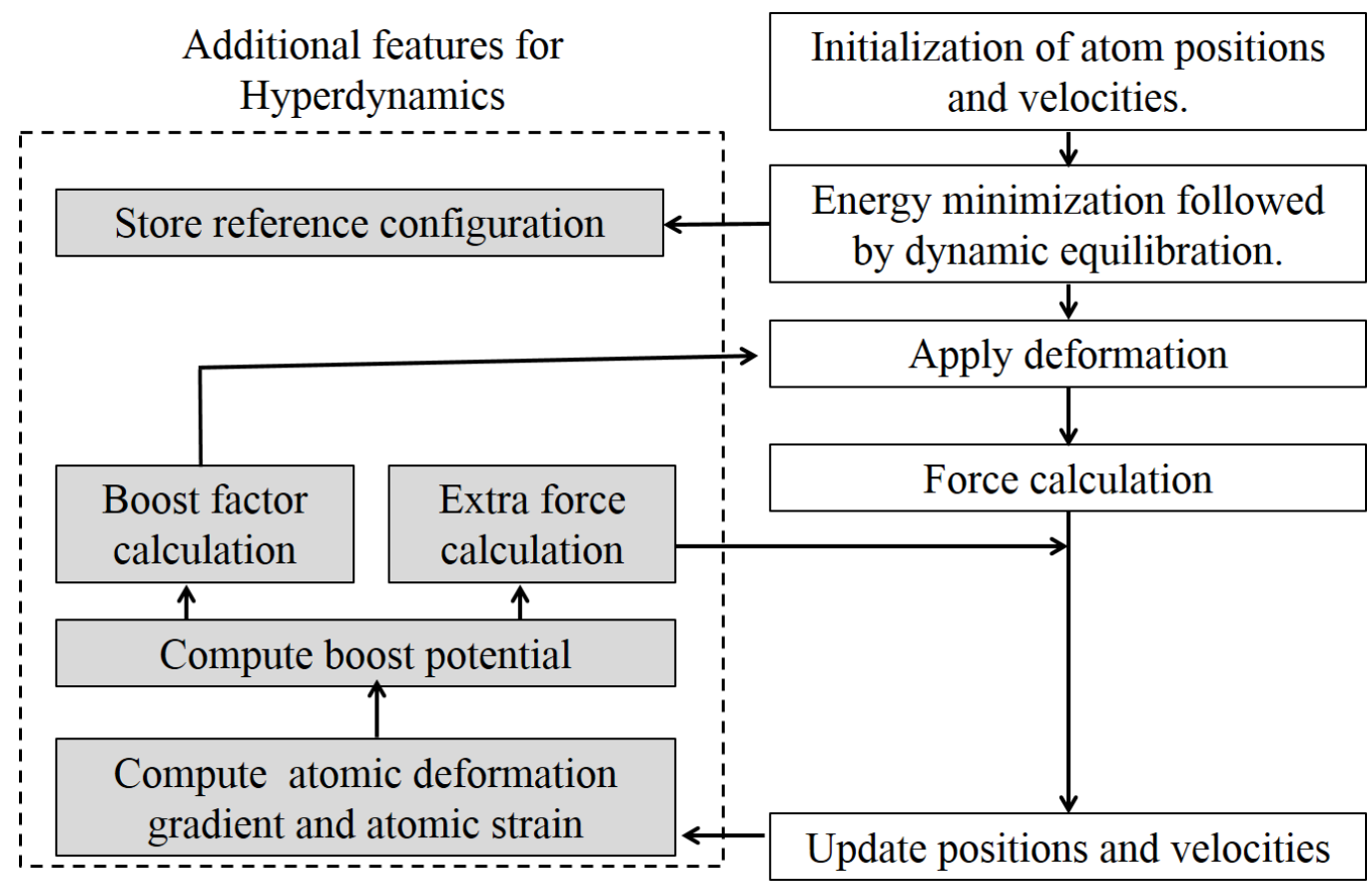

Figure 6: A flowchart showing the strain-boost hyperdynamics implementation in LAMMPS. Tasks inside the dashed box are added for the hyperdynamics acceleration in conjunction with the conventional MD operations.

The process of strain-boost hyperdynamics implementation in LAMMPS is briefly discussed in this section with a schematic depiction in figure 6 . The atomistic simulation specimen is created by filling the sample geometry with atoms conforming to the crystallographic lattice structure of the material in consideration. For investigating crack tip plasticity, an atomic crack is placed 
symmetrically at the center of the specimen by removing few layers of atoms as shown in figure 5 . This is followed by static minimization of the total potential energy for equilibrium configuration of the system. Next, the initial velocity of atoms are specified, based on the Maxwell-Boltzmann distribution for the simulation temperature taken to be $300 \mathrm{~K}$ in this study. Dynamic equilibration using the Nose-Hoover thermostat [10, 22] is carried out to achieve traction free outer surfaces while maintaining the system temperature. The equilibrated atomic positions are stored as the reference configuration. This is needed for computing the deformation gradients and atomic strains in subsequent simulations. This reference configuration will be updated once nucleated dislocations glide to their stable configuration.

Following equilibration, the system is deformed by displacing individual atoms conforming to the affine transformation, thereby achieving an incremental ensemble strain of $\Delta \epsilon$, given as:

$$
\Delta \epsilon=\Delta t_{p h y} \dot{\epsilon}
$$

$\Delta t_{\text {phy }}$ is the physical time at which the atomic system evolves under the boost potential $\Delta V$. Following equation (3), it is related to the MD time step $\Delta t_{M D}$ as $\Delta t_{p h y}=\Delta t_{M D} \exp \left(\Delta V / K_{b} T\right)$. The displacement step is followed by the calculation of conventional forces due to the inter-atomic interaction and additional forces due to boost potential. The system is accordingly moved forward in time by integrating the equations of motion using a velocity-Verlet time integration scheme [32]. Once the positions and velocities are updated, the new atomic strain, total per atom force (inter-atomic interaction force + boost force) and boost factor $\left(\exp \left(\Delta V / K_{b} T\right)\right)$ per atom are respectively calculated. Subsequently, the system is deformed by an incremental strain of $\Delta \epsilon$ according to equation (23) and the process continues.

\subsection{Validation of the Strain-Boost Hyperdynamics Model with Conventional MD Results}

Prior to the use of strain-boost hyperdynamics based MD simulation for low strain-rate deformation analysis, it is important to validate the model with results from conventional MD simulations. To accomplish this, hyperdynamicsbased accelerated MD and conventional MD simulations are conducted for an atomistic model of single crystal nickel specimen. The atomic simulation specimen has physical dimensions of $100 \mathrm{~nm} \times 50 \mathrm{~nm} \times 25 \mathrm{~nm}$, which consists 
of $\sim 10$ million atoms. The orientation of the crystal is $x \rightarrow[110], y \rightarrow[111]$, $z \rightarrow[11 \overline{2}]$. A through thickness uniform crack of width $10 \mathrm{~nm}$ is embedded in the specimen. Two different strain-rates, viz. $10^{7} \mathrm{~s}^{-1}$ and $10^{8} \mathrm{~s}^{-1}$, are applied to the simulations for comparison. The resulting macroscopic stressstrain response and evolution of dislocation density are shown in figure 7 . The critical nucleation stress and the evolution of different dislocation densities e.g. mobile and immobile dislocations by the two methods are in good agreement. It is noteworthy that hyperdynamics is most effective when the events of interest are very infrequent, which makes the achievable boost to be dependent on temperature. At higher temperatures, the occurrence of infrequent events is aided by higher kinetic energy of individual atoms that in turn reduces the achievable boost.

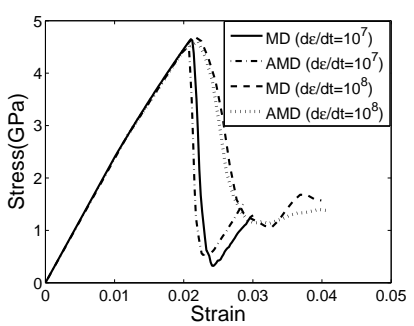

(a)

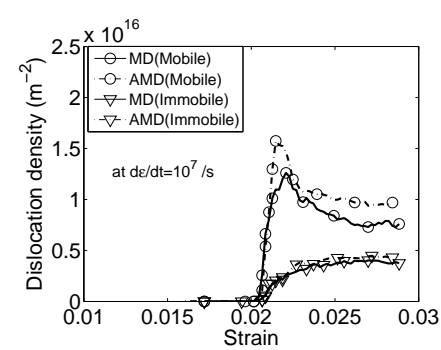

(b)

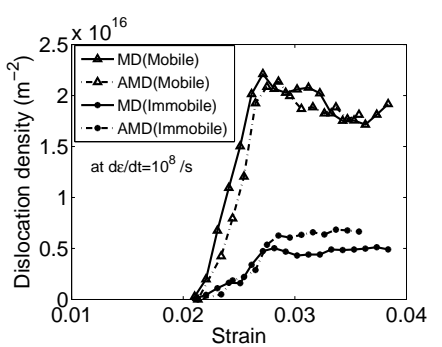

(c)

Figure 7: Validation of the strain-boost hyperdynamics-based MD model with conventional MD results: Comparisons of (a) volume averaged stress and (b,c)dislocation densities, as a function of the macroscopic strain.

To investigate the temperature and strain-rate dependence on achievable boost, similar computational experiments are performed for different strain-rates viz. $10^{9}, 10^{8}$ and $10^{7}$ and temperatures viz. $100 \mathrm{~K}$ and $300 \mathrm{~K}$. The achieved average strain-boost is calculated by monitoring the CPU time taken by the two methods. To extend the achievable boost prediction for lower strain-rates of $\sim 10^{4}$, hyperdynamics-based MD simulations are performed to get the first nucleation event. These rates are beyond the reach of conventional MD with most current computational platforms. With knowledge of the critical strain for nucleation for a particular strain-rate and temperature, the total number of MD steps that would have been needed for conventional MD can be approximately calculated. The ratio of time steps 
with conventional MD to that with hyperdynamics, multiplied by an adjustment factor to take care of the additional computation in hyperdynamics gives an idea of the achievable boost. Figure 8 shows an exponential increase of this achievable boost with decreasing strain-rates. This is a desired benefit of accelerated MD simulations.

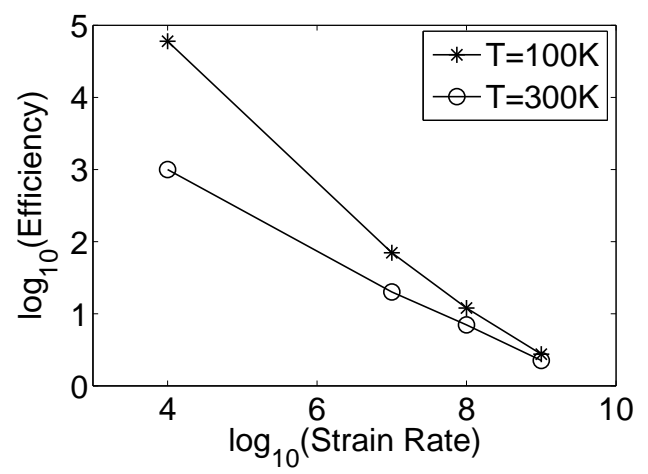

Figure 8: Efficiency of the hyperdynamics-based MD model for different strain-rates and temperatures.

\section{Numerical Simulation of a Crystalline Material with an Embed- ded Crack}

The examples in this section revisit the characterization and quantification of atomic-level deformation mechanisms obtained by conventional MD simulations of a crystalline material with an embedded crack in [39]. The strain-boost hyperdynamics based accelerated MD simulations are used to compare the deformation mechanisms at the high strain-rates similar to those in [39]. On the other hand, simulations at lower strain-rates are examined for the effectiveness of predictions based on high strain rate MD simulations.

\subsection{MD model of a Single Crystal Nickel with an Embedded Crack}

The geometric features and loading characteristics of a single crystal nickel specimen with an embedded crack are shown in figure 9. A similar model has been used in [39], where it was established that a simulation box size of $80 \mathrm{~nm} \times 44 \mathrm{~nm} \times 25 \mathrm{~nm}$ is sufficient for the boundary effects to be insignificant on crack-tip dislocation nucleation. In the present simulations, a box of size $100 \mathrm{~nm} \times 50 \mathrm{~nm} \times 25 \mathrm{~nm}$ is used. It consists of about 10 million 
atoms, the lattice constant of nickel being $3.52 A^{\circ}$.

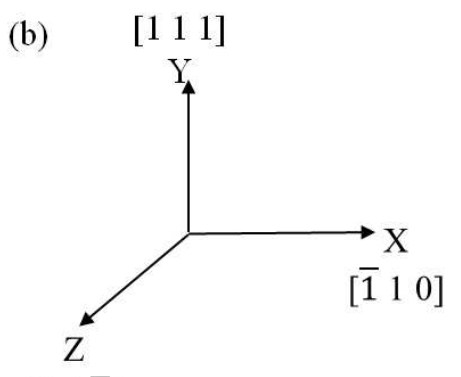

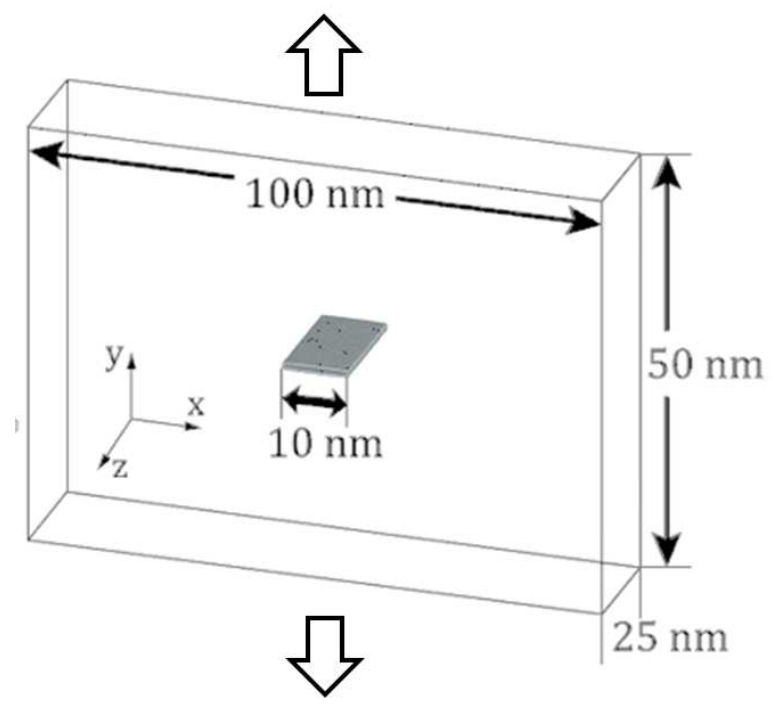

(a) $\left[\begin{array}{lll}1 & 1 & \overline{2}\end{array}\right]$

(c)

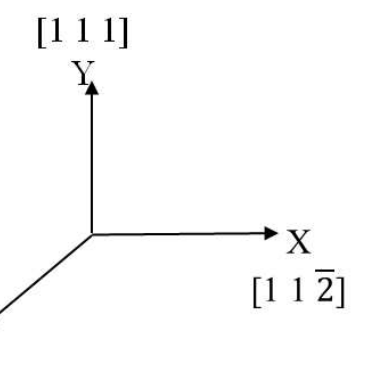

$\left[\begin{array}{lll}1 & 1 & 0\end{array}\right]$

Figure 9: Geometric details of the MD model with boundary conditions: (a) physical representation of the specimen with an embedded crack; crystal orientations with respect to specimen coordinate axes for: (b) orientation-1, and (c) orientation-2.

The interaction between nickel atoms in the MD simulations is computed by a many-body potential function, described by the embedded-atom method or EAM developed in [2]. This potential is appropriate for most transition metal systems. The functional form of the EAM potential energy of the $i-t h$ atom is given as:

$$
E_{i}=\mathcal{F}_{\alpha}\left(\sum_{i \neq j} \rho_{\beta}\left(r_{i j}\right)\right)+\frac{1}{2} \sum_{j=1, j \neq i}^{N} \phi_{i j}\left(r_{i j}\right)
$$

The first term is an embedding function that represents the energy required to place an atom $i$ of type $\alpha$ into the electron cloud. The electron cloud density is a summation over many atoms, usually limited by a cutoff radius. In the embedding energy function $\mathcal{F}_{\alpha}, \rho_{\beta}$ is the contribution to the electron 
charge density from atom $j$ of type $\beta$ at the location of atom $i$. It is a function of the distance $r_{i j}$ between atoms $i$ and $j$ within a cutoff distance. The second term is a short-range electrostatic pair-wise potential as a function of the distance $r_{i j}$ between atoms $i$ and $j$. For a single element system, three scalar functions must be specified, viz. the embedding function, a pair-wise interaction function, and the electron cloud contribution function.

Periodic boundary conditions are applied to eliminate free surface effects. Translational symmetry extends the crack infinitely in the $z$-direction. The simulation box is subjected to an applied $y$-direction displacement that governs a controlled incremental strain of $\Delta \epsilon$ in equation (23). The incremental strain is realized by displacing atoms via affine transformation prior to time integration of the governing equations.

Simulations with the accelerated MD model are used to understand deformation mechanisms at the crack tip and to quantify state variables that govern plastic deformation, viz. evolution of mobile and immobile dislocation densities, twin volume fraction etc., at strain-rates that are much lower compared to what are achievable in conventional MD. Various methods have been suggested in [39] to extract deformation mechanisms from simulated atomic

configurations. The DXA method of extracting the topological structure of dislocations [28] and the atomic-scale deformation gradient measurement [42] for identifying twinned regions are utilized in this work.

\subsection{Results of Hyperdynamics MD Simulations}

MD simulations are conducted for two different lattice orientations, corresponding to the following directions for the $x-y-z$ axes in figure 9 (a). They are:

1. Lattice Orientation $1: x \rightarrow[\overline{1} 10], y \rightarrow[111], z \rightarrow[11 \overline{2}]$

2. Lattice Orientation $2: x \rightarrow[11 \overline{2}], y \rightarrow[111], z \rightarrow[1 \overline{1} 0]$

The significance of these two orientations is that the three slip planes (111), $(1 \overline{1} 1)$ and $(11 \overline{1})$ have the same in-plane stresses when the sample is loaded in the $y$-direction, which is the crystallographic [111] direction. In contrast, the (111) plane, being perpendicular to the $y$--axis, has zero in-plane stresses. Different orientations of the embedded crack with respect to the crystallographic axis leads to the activation of slip in different directions. This in 
turn leads to the generation of different kinds of dislocations governing plastic behavior.

The two orientations have also been used in [39] with conventional MD, at an applied strain-rate of $2 * 10^{7} / \mathrm{sec}$, to investigate orientation dependence of crack tip plasticity. However at this high strain-rate, the atomic system does not have enough time for activation of thermal activation dependent mechanisms. Therefore, the simulation temperature in [39] was kept to $2 \mathrm{~K}$ and only stress driven phenomenon was studied. With the hyperdynamics based accelerated MD in the present study, a strain-rate of $10^{4} / \mathrm{sec}$ is achieved. Hence, both the stress-driven and thermally activated processes can be accurately represented, for which the system temperature is kept at $300 \mathrm{~K}$.

\subsubsection{Results for lattice orientation-1}

As mentioned earlier, for lattice orientation-1, three slip planes have the same in-plane stress and the crack tip extends in crystallographic [11 $\overline{2}]$ direction. Figure 10a shows the evolution of macroscopic volume-averaged stress $\sigma_{y y}$ as a function of the $y$ - direction engineering strain $\epsilon_{y y}$. In the tensile stress-strain curve, the peak stress corresponds to the critical resolved shear stress required for nucleating the dislocation. The stress subsequently decreases due to the motion of dislocation, which releases the elastic strain in the system. The results are for two strain-rates, viz. $2 * 10^{7} / \mathrm{sec}$ with conventional MD and $10^{4} /$ sec with hyperdynamics. The critical stress, which corresponds to nucleation of first leading partial from the crack tip, are respectively $\sim 4.65 \mathrm{GPa}$ and $\sim 4.38 \mathrm{GPa}$ for the strain-rates $2 * 10^{7} / \mathrm{sec}$ and $10^{4} /$ sec. The drop in critical stress is due to the fact that at finite temperatures and lower strain-rate, thermal activation plays a major role in the nucleation process. The evolution of dislocations is also investigated to understand the effect of strain-rate in more details. Figure 10b shows the evolution of total, mobile (Shockley partial) and immobile (stair-rod) dislocation density as the specimen deforms plastically. The dislocation density is approximated as the total dislocation length per unit volume. At a strain-rate of $10^{4} / \mathrm{sec}$, the stabilized mobile dislocation density is almost $50 \%$ higher than that at $2 * 10^{7} / \mathrm{sec}$. Higher mobile dislocation density implies more ductility in the plastic regime. This difference in dislocation density for different strain-rates is due to the fact that at lower strain-rates, the system nucleates lesser number of dislocations as shown in figure 11. This enhances the 
free path for dislocations to glide before interacting with other dislocations from intersecting slip planes. Contrary to mobile dislocation density, which is much higher at low strain-rate compared to higher rates, the immobile dislocation density is less at lower strain-rates. The difference in immobile dislocation density for the two strain-rates are not so significant. The presence of immobile dislocations hardens the material by making it difficult for mobile dislocations to glide freely. At lower strain-rates, the combined effect of higher mobile dislocation density and lower immobile dislocation density makes the material to hardens slowly. This is evident from the reduced flow stress of the material.

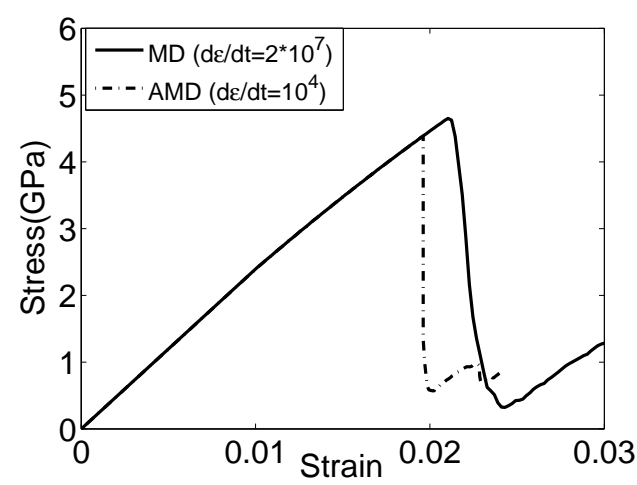

(a)

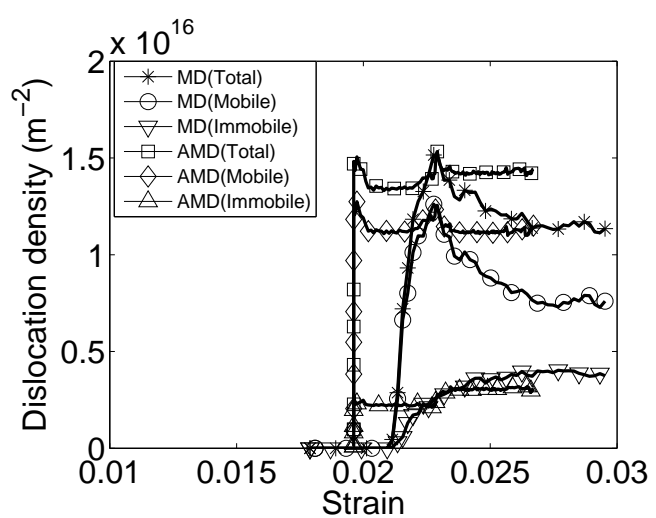

(b)

Figure 10: Evolution of variables for the crystal lattice orientation-1 by conventional MD and accelerated MD (AMD): (a) average tensile stress, and (b) total, mobile and immobile dislocation densities as a function of increasing tensile strain.

\subsubsection{Results for lattice orientation-2}

For the crystal lattice orientation-2, the crack tip extends in crystallographic $[1 \overline{1} 0]$ direction, which makes the $(11 \overline{1})$ plane parallel to the crack tip. In this particular orientation, the $[11 \overline{2}]$ direction corresponds to the maximum in-plane stress, which favors nucleation of leading partial dislocations. A leading partial with Burgers vector $b=\frac{1}{6}[11 \overline{2}]$ is emitted irrespective of the strain-rate as shown in figure 13(a). This dislocation is purely edge in character. At the high strain-rate of $2 * 10^{7} / \mathrm{sec}$, this leading partial is followed by another leading partial in an adjacent parallel slip plane as shown in figure 13(b). This combination forms a micro-twin band. The twinned re- 
(a)

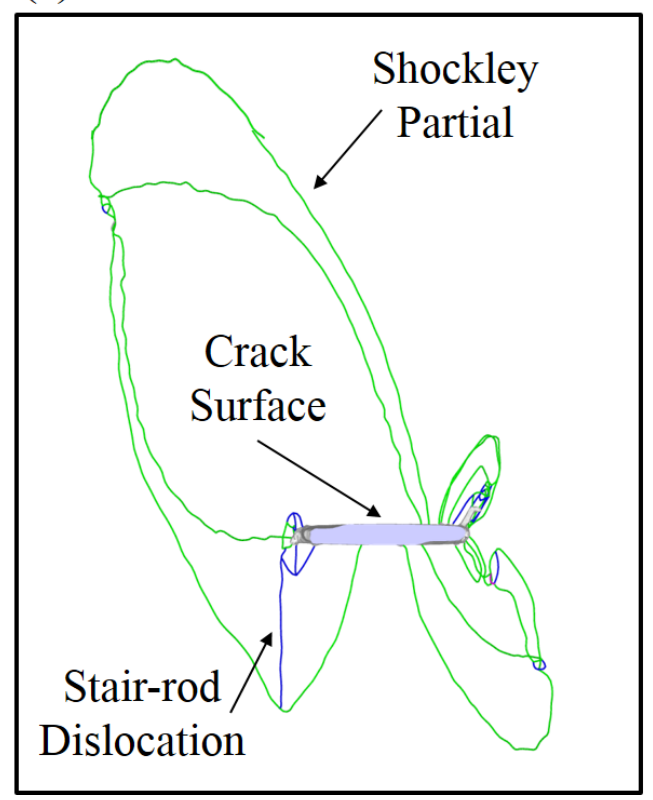

(b)

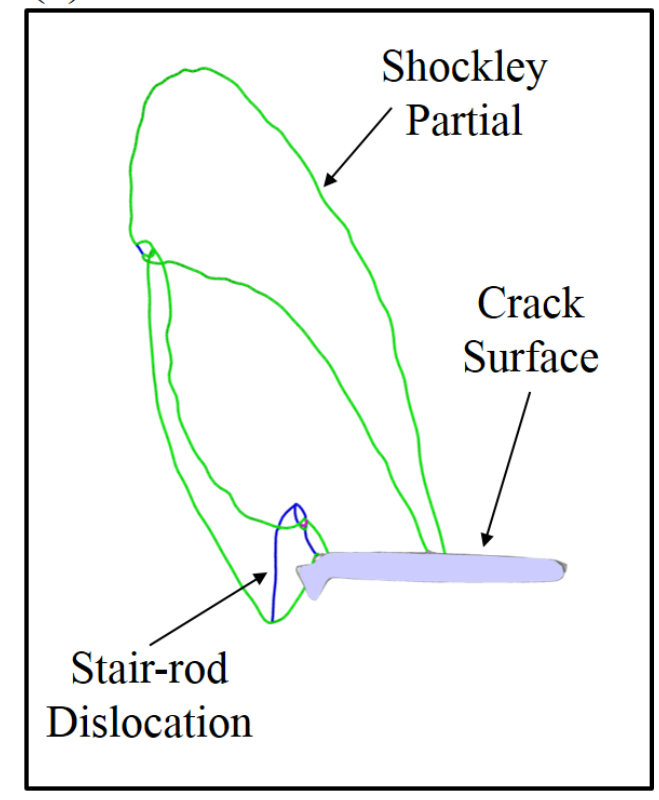

Figure 11: Initial crack-tip dislocation segments for orientation-1 at strain-rates of (a) $2 * 10^{7} /$ sec by conventional MD, and (b) $10^{4} /$ sec by accelerated MD. 
gion increases by migration of twin boundaries due to subsequent nucleation of leading partials in parallel slip planes. However for the lower strain-rate of $10^{4} / \mathrm{sec}$, the leading partial is followed by a trailing partial. Together they form a full dislocation as seen in figure 13(c). This significant difference in deformation mechanisms is clearly a strain rate-effect. It also has a profound effect on the macroscopic stress-strain response of the material as depicted in figure 12. A full dislocation i.e a leading partial followed by a trailing partial in the same slip plane, does not leave behind any stacking fault and once a full dislocation nucleates it carries plastic strain by gliding along the slip plane. On the other hand for twin, plastic strain is carried by migration of twin boundary which is accomplished by nucleation of successive leading partial in parallel slip planes. Since the gliding stress is much smaller than the nucleation stress, it makes full dislocation a more superior carrier of plastic strain away from crack tip comparison to twin, which is also evident from macroscopic stress-strain plot. Present finding is in agreement with [36] where a transition from twin (under short time and high load) to full dislocation (under long time and smaller load) has also been observed under a fixed applied stress intensity $\left(K_{I}\right)$. At this point it is worthy to mention that for present crack orientation and with mode-I loading, nucleation of twin or full dislocation could also depend on the atomic configuration of the crack tip. For some particular crack-tip atomic configuration plastic deformation is dislocation dominated at both low and high applied strain rate. This suggests that to develop an accurate analytic model predicting the critical strain rate corresponding to twin to dislocation transition may need to take local crack tip atomic configuration into consideration.

\section{Concluding Remarks}

This paper develops a strain-boost hyperdynamics based accelerated molecular dynamics framework for modeling deformation in crystalline materials with an embedded crack. MD simulations are capable of depicting evolving deformation mechanisms in materials at the atomic scale. They have been used for continuum-scale material property assessment by hierarchical and concurrent coupling with continuum models. A major limitation of such coupling with conventional MD simulations is that very small MD time-scales restrict the achievable strain-rates to be much higher than experimentally observed rates, needed for continuum scale modeling, e.g. using crystal plas-

ticity finite element methods. An approach that has been used to extract 


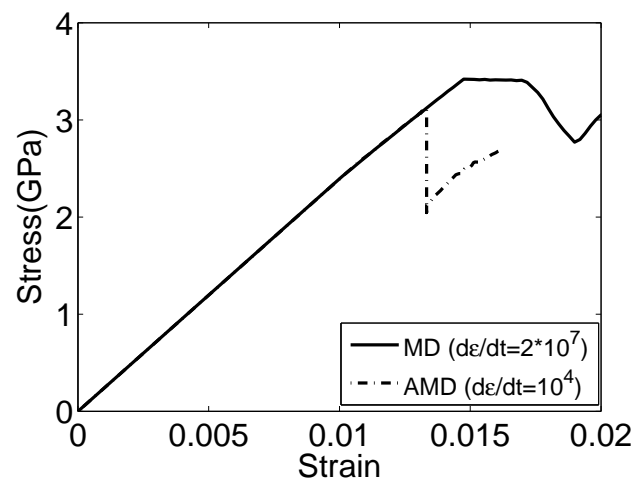

Figure 12: Evolution of macroscopic stress as a function of engineering strain for orientation- 2 by conventional MD and accelerated MD (AMD).

low strain-rates by simple extrapolation of the higher strain-rate results. While this approach may be reasonable when the deformation mechanisms remain unchanged over long temporal domains, their predictive capabilities are severely compromised in the presence of multiple competing mechanisms, whose activation depends on the deformation rate itself. Furthermore, in a concurrent coupling of MD with continuum models, stability requirements of the integration algorithm can adversely affect time increments in the continuum model. The hyperdynamics accelerated MD tool that is adopted and developed here is demonstrated to overcome these limitations and achieve experimentally observed strain rates.

The hyperdynamics method is based on lifting the basin of the potentialwell and biasing the local potential landscape in the MD model. The transition state theory is used to relate the evolution of the biased system with the unbiased system. The strain-boost hyperdynamics method uses the second invariant of the deviatoric atomic strain to construct the boost potential resulting in better nearest-neighbor atomic shell than the bond length alone. This method is incorporated in the LAMMPS code and validated for an atomistic model of a single crystal nickel specimen. Excellent agreement is achieved with high strain-boost over conventional MD. High effectiveness is achieved for low frequency of events of interest that makes the achievable boost to be dependent on the temperature. Strain rates of the order of $10^{4}$ are simulated with this method with an exponential increase in the achievable boost with decreasing strain-rates. 


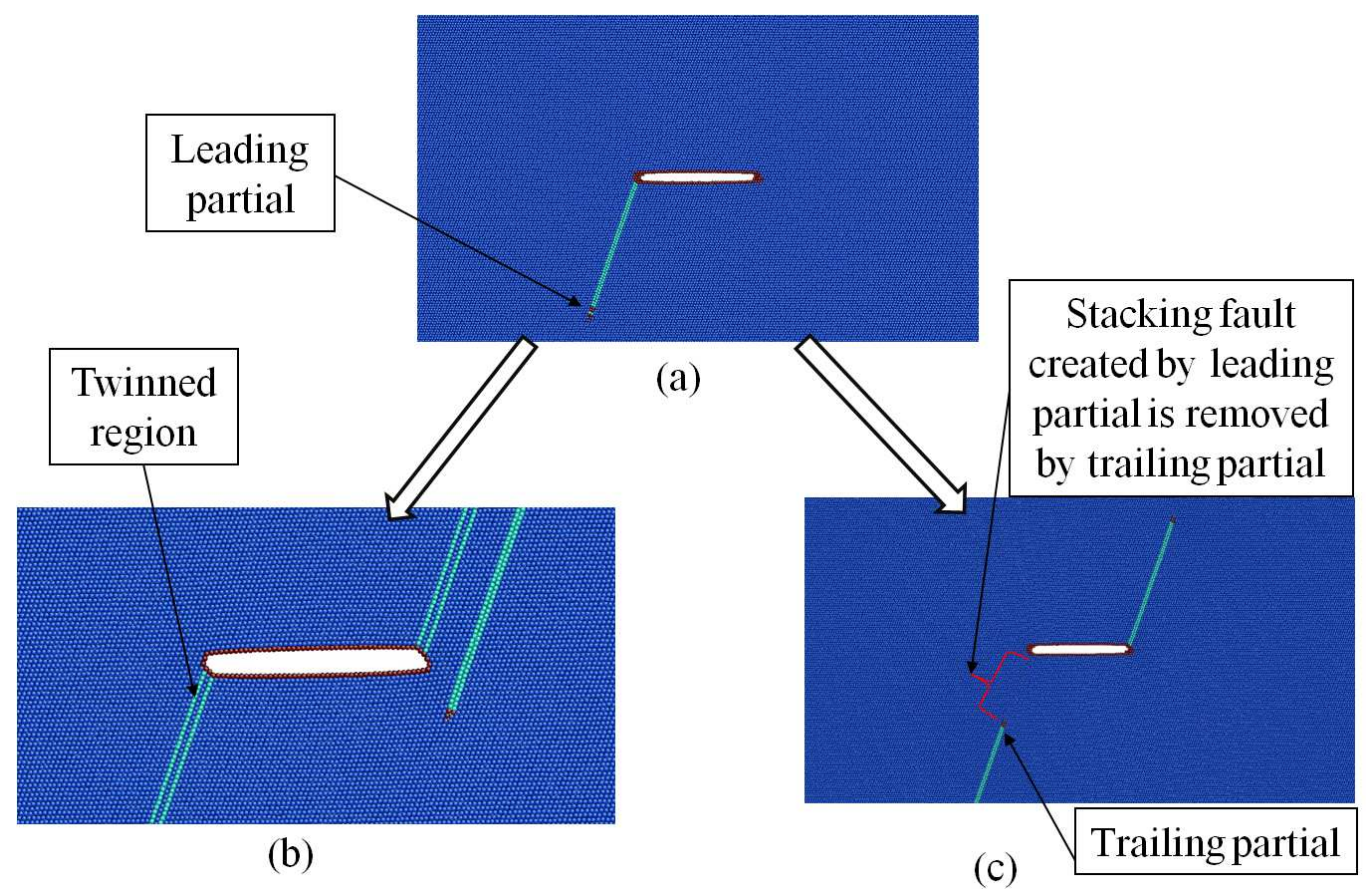

Figure 13: Snapshots of plastically deformed atomic configuration in orientation-2, ploted using AtomEye [19] with color based on common neighbor analysis (CNA [9]), showing deformation mechanisms at different strain-rates: (a) leading partial nucleation at the onset of plasticity irrespective of strain rate; (b) leading partial followed by another leading partial in the parallel slip plane (twining partial) at high strain-rates, and (c) leading partial is followed by a trailing partial at lower strain-rates.

Numerical studies are subsequently conducted for a single crystal nickel material with an embedded crack. In contrast to observations at high strainrates of the order of $\sim 10^{7}$ in [39], this study is intended to characterize evolving mechanisms at lower strain rates, e.g. $\sim 10^{4}$. MD simulations are conducted for two different lattice orientations or Schmid factors. Different orientations of the embedded crack with respect to the crystallographic axis leads to slip activation in different directions causing nucleation of different kinds of dislocations. For the first orientation, the mobile dislocation density remains nearly constant with periodic burst of dislocations. This is a typical feature of plastic deformation in single crystals, as has been observed for aluminum single crystals in [23]. For the second orientation, the plastic deformation mechanism is dislocation dominated for the lower strain-rate, as 
opposed to twin dominated prediction at high strain-rate with conventional MD. This observation is also consistent with the experimental observation that Nickel shows deformation twinning only under shock loading at low temperatures $[7,21]$. In summary, the present study shows that the strain-boost hyperdynamics is an effective method to overcome the time scale limitation of conventional MD for continuum modeling of elastic-plastic phenomena. Such methods are important for lower strain-rate, coupled continuum-atomistic simulation methods that exhibit multiple time-scales in the disparate domains.

\section{ACKNOWLEDGMENTS}

This work has been supported by National Science Foundation, Mechanics ans Structure of Materials Program through grant No. CMMI-1200231 (Program Manager: Dr. Clark Cooper and Dr. Alexis Lewis). This sponsorship is gratefully acknowledged.

\section{References}

[1] I. Akio, S. Ogata, H. Kimizuka, and J. Li. Adaptive-boost molecular dynamics simulation of carbon diffusion in iron. Phys. Rev. B., 85: 064303, 2012. doi: http://dx.doi.org/10.1103/PhysRevB.85.064303.

[2] J.E. Angelo, N.R. Moodym, and M.I. Baskes. Trapping of hydrogen to lattice defects in nickel. Modelling Simul. Mater. Sci. Eng., 3(3): 289-307, 1995. doi: http://dx.doi.org/10.1088/0965-0393/5/6/007.

[3] K. L. Baker and D. H. Warner. Extended timescale atomistic modelling of crack tip behavior in aluminum. Modelling and Simul. Mater. Sci. Eng., 20:065005, 2012. doi: http://dx.doi.org/10.1088/09650393/20/6/065005.

[4] B. A. Berg and T. Neuhaus. Multicanonical ensemble : A new approach to simulate first-order phase transitions. Phys. Rev. Lett., 156:472-477, 1992.

[5] E. A. Carter, G. Ciccotti, J. T. Hynes, and R. Kapral. Constrained reaction coordinate dynamics for the simulation of rare events. Chem. Phys. Lett., 156:472-477, 1989. 
[6] H. Eyring. The activated complex in chemical reactions. The Journal of Chemical Physics., 3:107, 1935. doi: http://dx.doi.org/10.1063/1.1749604.

[7] P. Haasen. Plastic deformation of nickel single crystals at low temperatures. Phil. Mag., 3:384-418, 1958 . doi: http://dx.doi.org/10.1080/14786435808236826.

[8] S. Hara and J. Li. Adaptive strain-boost hyperdynamics simulations of stress-driven atomic processes. Phys. Rev. B., 82:184114, 2010. doi: http://dx.doi.org/10.1103/PhysRevB.82.184114.

[9] D.J. Honeycutt and H.C. Anderson. Molecular dynamics study of melting and freezign of small lennard-jones clusters. J. Chem. Phys., 91: 4950-4963, 1987. doi: http://dx.doi.org/10.1021/j100303a014.

[10] W. G. Hoover. Canonical dynamics: Equilibrium phase-space distributions. Phys. Rev. A, 31(3):16951697, $1985 . \quad$ doi: http://dx.doi.org/10.1103/PhysRevA.31.1695.

[11] H. Huang, D. Perez, and A. F. Voter. Hyperdynamics boostfactor achievable with an ideal bias potential. The Journal of Chemical Physics, 143:074113, 2015. doi: http://dx.doi.org/10.1063/1.4928636.

[12] K. Kadau, T.C. Germann, and P.S. Lomdahl. Molecular dynamics comes of age: 320-billion-atom simulation on bluegene/l. Int. J. Mod. Phys. C, 17:1755-1761, 2006.

[13] J. Kastner. Umbrella integration in two or more reaction coordinates. The Journal of Chemical Physics., 131:034109, 2009. doi: http://dx.doi.org/10.1063/1.3175798.

[14] J. Kastner. Umbrella sampling. WIREs Comput Mol Sci., 1:932-942, 2011. doi: http://dx.doi.org/10.1002/wcms.66.

[15] W. K. Kim and M. L. Falk. Accelerated molecular dynamics simulation of low-velocity frictional sliding. Modelling and Simul. Mater. Sci. Eng., 18:034003, 2010. doi: http://dx.doi.org/10.1088/09650393/18/3/034003. 
[16] W. K. Kim and M. L. Falk. A practical perspective on the implementation of hyperdynamics for accelerated simulation. The Journal of Chemical Physics., 140:044107, 2014. doi: http://dx.doi.org/10.1063/1.4862269.

[17] A. Laio and F. L. Gervasio. Metadynamics:a method to simulate rare events and reconstruct the free energy in biophysics, chemistry and material sciencea. Rep. Prog. Phys., 71:126601, 2008. doi: http://dx.doi.org/10.1088/0034-4885/71/12/126601.

[18] A. Laio and M. Parrinello. Escaping free-energy minima. PNAS., 99: 20, 2002. doi: http://www.pnas.org/content/99/20/12562.

[19] J. Li. Atomeye: an efficient atomistic configuration viewer. Modelling Simul. Mater. Sci. Eng., 11:173-177, $2003 . \quad$ doi: http://dx.doi.org/10.1088/0965-0393/11/2/305.

[20] A. Miron and K. A. Fichthorn. Accelerated molecular dynamics with the bond-boost method. The Journal of Chemical Physics, 119:12, 2003. doi: http://dx.doi.org/10.1063/1.1603722.

[21] L. Murr and E. Esquivel. Observations of common microstructural issues associated with dynamic deformation phenomena: Twins, microbands, grain size effects, shear bands, and dynamic recrystallization. J. Mater. Sci., 39:1153-1168, 2004. doi: http://dx.doi.org/10.1023/B:JMSC.0000013870.09241.c0.

[22] S. Nose. A unified formulation of the constant temperature moleculardynamics methods. Jour. Chem. Physics, 81(1):511519, 1984 . doi: http://dx.doi.org/10.1063/1.447334.

[23] Sang Ho Oh, Marc Legros, Daniel Kiener, and Gerhard dehm. In situ observation of dislocation nucleation and escape in a submicrometre aluminium single crystal. Nature Materials, 8:95-100, 2009. doi: http://dx.doi.org/10.1038/NMAT2370.

[24] D. Perez and A. F. Voter. Accelerating atomistic simulations through self-learning bond-boost hyperdynamics. LA-UR-08-5519, 2008. 
[25] D. Perez, B. P. Uberuaga, Y Shim, J. G. Amar, and A. F. Voter. Accelerated molecular dynamics methods: introduction and recent developments. Annual Reports in Computational Chemistry, Elsevier, 5:15741400, 2009. doi: http://dx.doi.org/10.1016/S1574-1400(09)00504-0.

[26] S.J. Plimpton. Fast parallel algorithms for short-range molecular dynamics. J. Comput. Phys., 117:1-19, 1995. doi: http://dx.doi.org/10.1006/jcph.1995.1039.

[27] M. R. Sorensen and A. F. Voter. Temperature-accelerated dynamics for simulation of infrequent events. The Journal of Chemical Physics, 112: 9599, 2000. doi: http://dx.doi.org/10.1063/1.481576.

[28] A. Stukowski and K. Albe. Extracting dislocations and non-dislocation crystal defects from atomistic simulation data. Modelling Simul. Mater. Sci. Eng., 18:085001, 2010. doi: http://dx.doi.org/10.1088/09650393/18/8/085001.

[29] D. G. Truhlar and B. C. Garrett. Variational transition-state theory. Acc. Chem. Res., 13:440-448, 1980.

[30] B. P. Uberusga, S. J. Start, and A. F. Voter. Parallel replica dynamics for driven systems: derivation and application to strained nanotubes. Phys. Rev. B., 75:014301, 2007. doi: http://dx.doi.org/10.1103/PhysRevB.75.014301.

[31] E. Vanden-Eijnden and F. A. Tal. Transition state theory: Variational formulation, dynamical corrections and error estimates. The Journal of Chemical Physics., 123:184103, 2005. doi: http://dx.doi.org/10.1063/1.2102898.

[32] L. Verlet. Computer "Experiments" on classical fluids. I. thermodynamical properties of lennard-jones molecules. Phys. Rev., 159(1):98-103, 1967. doi: http://dx.doi.org/10.1103/PhysRev.159.98.

[33] A. F. Voter. A method for accelerating the molecular dynamics simulation of infrequent events. The Journal of Chemical Physics., 106:4665, 1997. doi: http://dx.doi.org/10.1063/1.473503. 
[34] A. F. Voter. Hyperdynamics: Accelerated molecular dynamics of infrequent events. Phys. Rev. Lett., 78:20, 1997. doi: http://dx.doi.org/10.1103/PhysRevLett.78.3908.

[35] A. F. Voter. Parallel replica method for dynamics of infrequent events. Phys. Rev. B., 57:22, 1998. doi: http://dx.doi.org/10.1103/PhysRevB.57.R13985.

[36] D. H. Warner, W. A. Curtin, and S. Qu. Rate dependence of crack-tip processes predicts twining trends in f.c.c metals. nature materials, 6: 876-881, 2007. doi: http://dx.doi.org/10.1038/nmat2030.

[37] V. Yamakov, D. Wolf, S. R. Phillpot, A. K. Mukherjee, and H. Gleiter. Dislocation processes in the deformation of nanocrystalline aluminium by molecular-dynamics simulation. nature materials, 1:45-49, 2002. doi: http://dx.doi.org/10.1038/nmat700.

[38] V. Yamakov, D. H. Warner, E. Zamora, Saether, W. A. Curtin, and E. H. Glaessgen. Investigation of crack tip dislocation emission in aluminum using multiscale molecular dynamics simulation and continuum modeling. J. Mech. Phys. Solids, 65:35-53, 2014 . doi: http://dx.doi.org/10.1016/j.jmps.2013.12.009.

[39] J. Zhang and S. Ghosh. Md based study and characterization of deformation mechanisms near a crack. J. Mech. Phys. Solids, 61:1670-1690, 2013. doi: http://dx.doi.org/10.1016/j.jmps.2013.04.004.

[40] S. J. Zhou, D. M. Beazley, P. S. Lomdahl, and B. L. Holian. Largescale molecular dynamics simulations of three-dimensional ductile failure. Phys. Rev. Lett., 78(3):479-482, 1996.

[41] T. Zhu, J. Li, A. Samanta, A. Leach, and K. Gall. Temperature and strain rate dependence of surface dislocation nucleation. Phys. Rev. Lett., 100:025502, 2008. doi: http://dx.doi.org/10.1103/PhysRevLett.100.025502.

[42] J. A. Zimmerman, D. J. Bammann, and H. Gao. Deformation gradient for continuum mechanical analysis of atomistic simulation. Int. J. Solids and Structures, 46:238-253, 2009. doi: http://dx.doi.org/10.1016/j.ijsolstr.2008.08.036. 
(a)

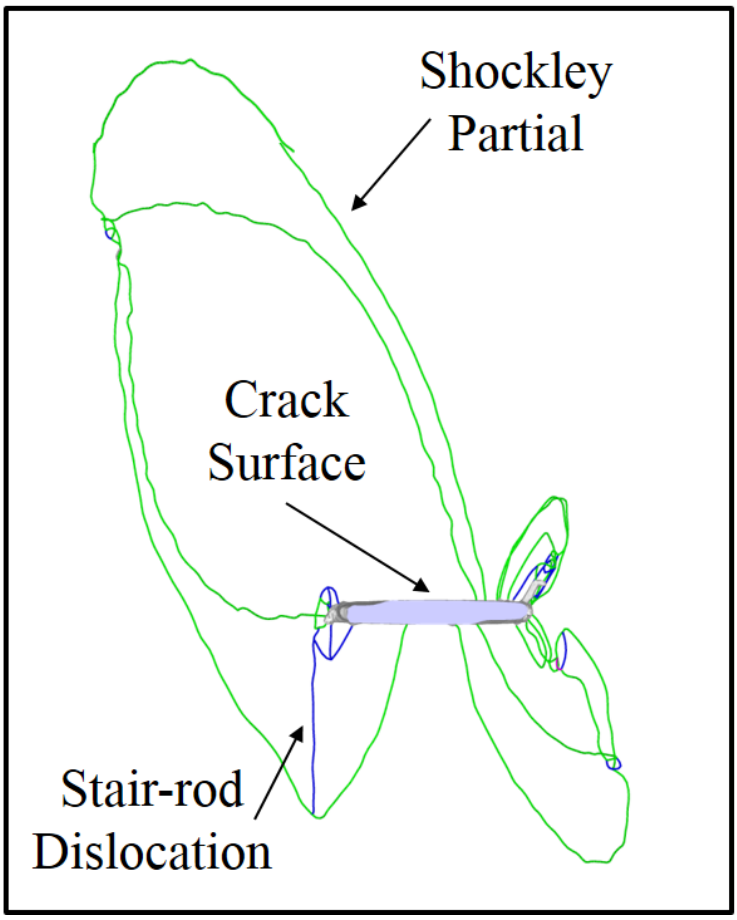

(b)

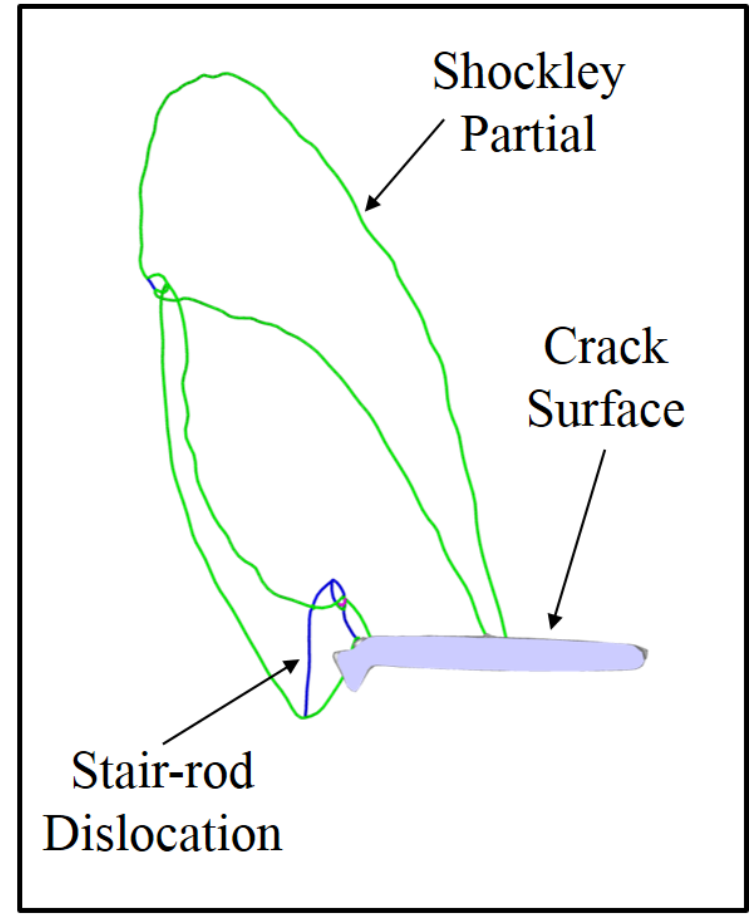

